\title{
On the Characterization of Expansion Maps for Self-Affine Tilings
}

\author{
Richard Kenyon • Boris Solomyak
}

Received: 16 September 2008 / Revised: 2 June 2009 / Accepted: 3 June 2009 /

Published online: 18 June 2009

(C) Springer Science+Business Media, LLC 2009

\begin{abstract}
We consider self-affine tilings in $\mathbb{R}^{n}$ with expansion matrix $\phi$ and address the question which matrices $\phi$ can arise this way. In one dimension, $\lambda$ is an expansion factor of a self-affine tiling if and only if $|\lambda|$ is a Perron number, by a result of Lind. In two dimensions, when $\phi$ is a similarity, we can speak of a complex expansion factor, and there is an analogous necessary condition, due to Thurston: if a complex $\lambda$ is an expansion factor of a self-similar tiling, then it is a complex Perron number. We establish a necessary condition for $\phi$ to be an expansion matrix for any $n$, assuming only that $\phi$ is diagonalizable over $\mathbb{C}$. We conjecture that this condition on $\phi$ is also sufficient for the existence of a self-affine tiling.
\end{abstract}

Keywords Self-affine tiling · Expansion map · Perron number

\section{Introduction}

Self-affine tilings arise in many different contexts, notably in dynamics (Markov partitions for hyperbolic maps [10, 15, 20]), logic (aperiodic tilings [14]), number theory (radix representations [12, 18]), physics (quasicrystals [3]), ergodic theory [21], and hyperbolic groups [4]. See [2, 19] for recent surveys with a large bibliography.

A self-affine tiling (SAT) $\mathcal{T}=\left\{T_{i}\right\}_{i \in I}$ of $\mathbb{R}^{n}$ is a covering of $\mathbb{R}^{n}$ with sets (tiles) $T_{i}$ satisfying the following properties:

The work of Kenyon was supported in part by NSERC and NSF. The research of Solomyak was supported in part by NSF grants DMS-0355187 and DMS-0654408.

R. Kenyon

Department of Mathematics, Brown University, Providence, RI 02912, USA

B. Solomyak $(\bowtie)$

Department of Mathematics, University of Washington, Box 354350, Seattle, WA 98195, USA

e-mail:solomyak@math.washington.edu 


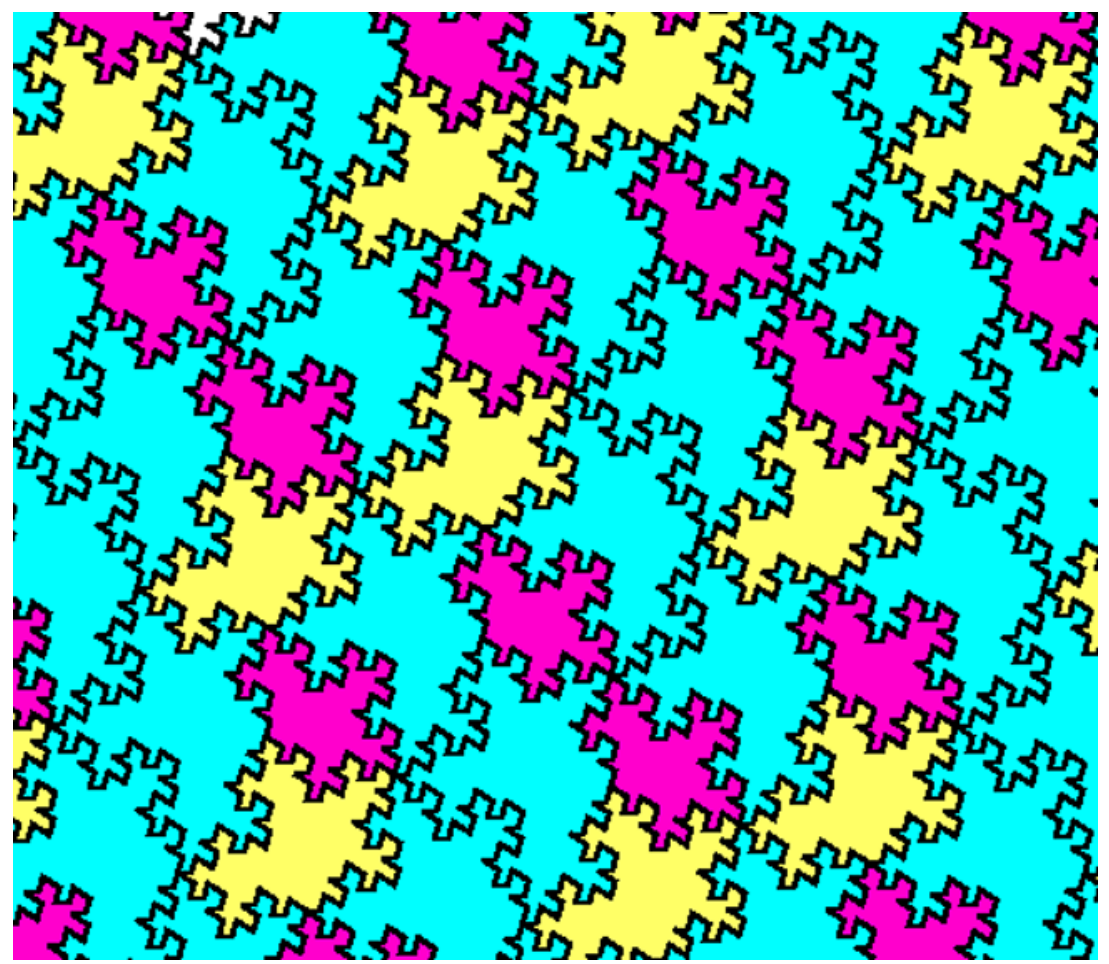

Fig. 1 A self-affine tiling in the plane with expansion $\phi(z)=\lambda z$ where $\lambda$ is the complex root of $x^{3}+x+1=0$. Here there are three tile types, all similar. The smallest scales to the medium which scales to the large; the large subdivides into a small and a large. One can construct this tiling using the method of [8, Sect. 6], as follows. To a reduced word in the free group on three letters $F(a, b, c)$ associate a polygonal path in $\mathbb{C}$ by sending $a^{ \pm 1}$ to $\pm 1, b^{ \pm 1}$ to $\pm \lambda, c^{ \pm 1}$ to $\pm \lambda^{2}$. Let $\psi$ be the endomorphism of $F(a, b, c)$ defined by $\psi(a)=b, \psi(b)=c, \psi(c)=a^{-1} b^{-1}$. Consider the three commutators $[a, b]=a b a^{-1} b^{-1},[b, c]$, and $[a, c]$; they represent three closed paths. Then $\lim _{n \rightarrow \infty} \lambda^{-n} \psi^{n}([a, c])$ is the boundary of the smallest tile; the other tiles boundaries are $\lim _{n \rightarrow \infty} \lambda^{-n} \psi^{n}([a, b])$ and $\lim _{n \rightarrow \infty} \lambda^{-n} \psi^{n}([b, c])$. The subdivision rule comes from the identities $\psi([a, c])=a^{-1}[a, b] a, \psi[a, b]=[b, c]$, and $\psi[b, c]=\left[c, a^{-1} b^{-1}\right]=\left(a^{-1}[a, c] a\right)\left(a^{-1} b^{-1}[b, c] b a\right)$

(1) Each tile $T_{i}$ is the closure of its interior.

(2) Interiors of tiles do not overlap.

(3) There are a finite number of tile types up to translation.

(4) The tiling is repetitive and has finitely many local configurations (see the next section for definitions).

(5) There is an expanding linear map $\phi: \mathbb{R}^{n} \rightarrow \mathbb{R}^{n}$ mapping tiles over tiles: the image of a tile $T_{i}$ is a union of tiles of $\mathcal{T}$, and two tiles of the same type have images which are translation-equivalent patches of tiles.

The simplest example is the periodic tiling with unit cubes and expansion mapping $\phi(x)=2 x$. However typically SATs are nonperiodic and have tiles with fractal boundaries. See Figs. 1 and 2 for examples in $\mathbb{R}^{2}$.

Lind [13] (using different language) gives a characterization of expansion factors of self-affine tilings in one dimension: $\lambda$ is the expansion of an SAT of $\mathbb{R}$ if and only 


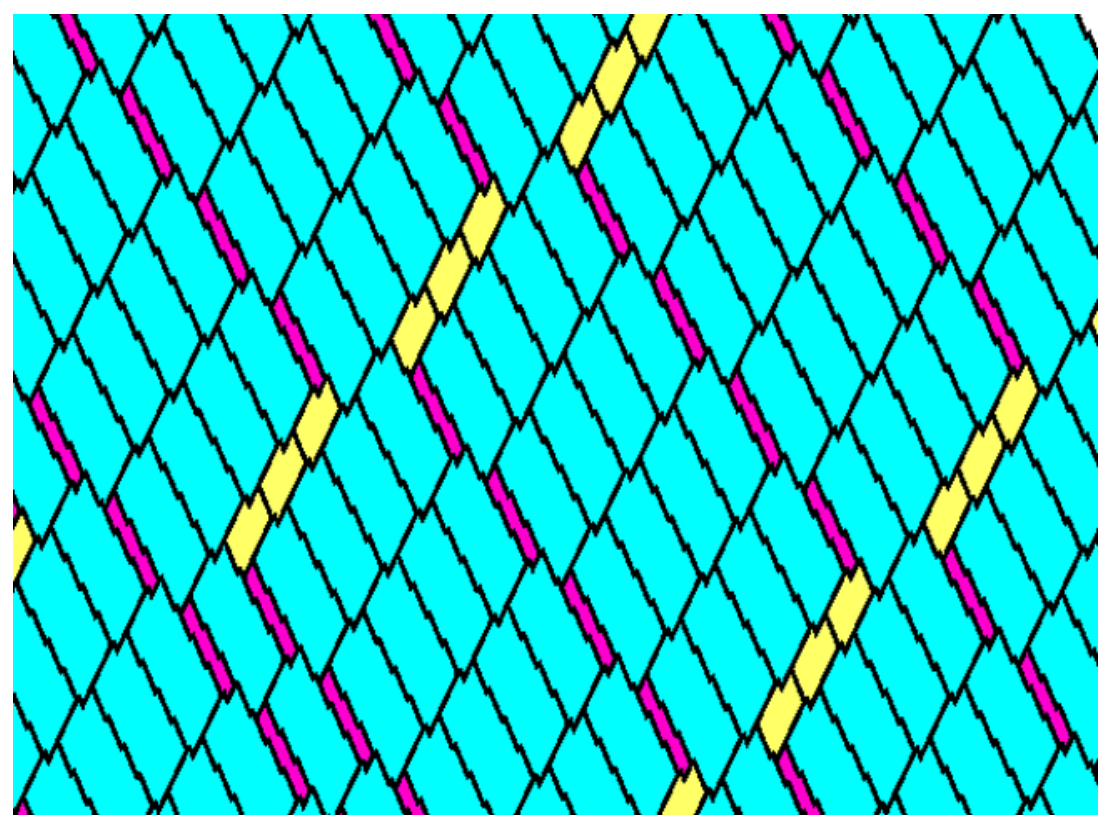

Fig. 2 A self-affine tiling in the plane with diagonal expansion matrix $\operatorname{Diag}\left[x_{1}, x_{2}\right]$ where $x_{1} \approx 2.19869$ and $x_{2} \approx-1.91223$ are roots of $x^{3}-x^{2}-4 x+3=0$

if $|\lambda|$ is a Perron number, that is, a real algebraic integer which is strictly larger in modulus than all of its Galois conjugates.

A self-affine tiling is self-similar if $\phi$ is a similarity (a homothety followed by a rotation). Thurston [23] showed that the expansion factor $\lambda \in \mathbb{C}$ of a self-similar tiling of $\mathbb{R}^{2}$ is a complex Perron number, that is, an algebraic integer which is strictly larger in modulus than its Galois conjugates except for its complex conjugate. In [8], a construction of a self-similar tiling for every complex Perron number is given; unfortunately, the proof as written in Sect. 4.5 of [8] is incomplete. A version of the construction does yield a tiling with expansion $\lambda^{k}$ for $k$ sufficiently large, and we hope that it can be modified to get a tiling with expansion $\lambda$, completing the characterization. This gap does not affect the construction in Sect. 6 of [8] which uses free group endomorphisms; however, the latter does not cover all the complex Perron numbers. See also [5] for a related construction.

In the current paper, we study SATs of $\mathbb{R}^{n}$ with expansion matrix $\phi$ which is diagonalizable over $\mathbb{C}$. We show that if $\phi$ is the expansion matrix for an SAT, then eigenvalues of $\phi$ are algebraic integers, and for every eigenvalue $\gamma$, all Galois conjugates of $\gamma$ which have modulus $\geq|\gamma|$ have multiplicity (among eigenvalues of $\phi$ ) at least as large as that of $\gamma$, see Theorem 3.1 below.

An alternative description of this criterion is that there is an integer matrix $M$ acting on $\mathbb{R}^{N}$ for some $N \geq n$, which has an invariant real subspace $W$ of dimension $n$, on which it has strictly larger growth (that is, strictly larger determinant, in absolute value) than for any other $n$-dimensional invariant subspace, and $M$ restricted to $W$ is linearly conjugate to $\phi$. 

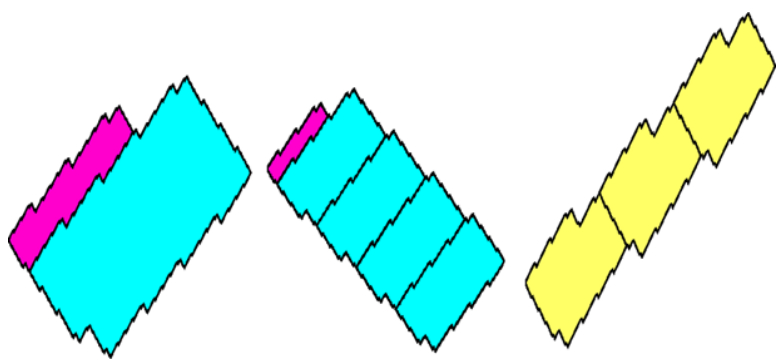

Fig. 3 Subdivision rule: $1 \rightarrow\{3,2\}, 2 \rightarrow\{3,2,2,2,2\}, 3 \rightarrow\{1,1,1\}$. The construction is similar to the previous example but with $a, b, c$ corresponding to vectors $(1,1),\left(x_{1}-1, x_{2}-1\right),\left(x_{1}^{2}-x_{1}, x_{2}^{2}-x_{2}\right)$ in $\mathbb{R}^{2}$, endomorphism $\psi(a)=a b, \psi(b)=c, \psi(c)=a b^{4}$ and tiles $[b, a],[b, c],[a, c]$

The converse to our result is open: does there exist, for every linear map $\phi$ satisfying the above conditions, an SAT with expansion $\phi$ ? We conjecture that the answer is yes.

In Fig. 2, we show an example of a self-affine (non-self-similar) SAT in the plane. The subdivision rule is indicated in Fig. 3.

Our methods do not at present extend to the nondiagonalizable case. However, we conjecture that the second description above holds in general, that is, without the constraint of diagonalizability, $\phi$ is the expansion of an SAT if and only if there is an integer matrix $M$ acting on $\mathbb{R}^{N}$ for some $N \geq n$, which has an invariant real subspace $W$ of dimension $n$, on which it has strictly larger growth (determinant) than for any other $n$-dimensional invariant subspace, and $M$ restricted to $W$ is linearly conjugate to $\phi$. For example, we conjecture that there is no SAT in $\mathbb{R}^{3}$ with expansion

$$
\left(\begin{array}{ccc}
3+\sqrt{2} & 1 & 0 \\
0 & 3+\sqrt{2} & 0 \\
0 & 0 & 3-\sqrt{2}
\end{array}\right),
$$

although it is easy to construct one with expansion

$$
\left(\begin{array}{ccc}
3+\sqrt{2} & 0 & 0 \\
0 & 3+\sqrt{2} & 0 \\
0 & 0 & 3-\sqrt{2}
\end{array}\right) .
$$

\section{Preliminaries}

We say that a tiling $\mathcal{T}=\left\{T_{i}\right\}_{i \in I}$ has a finite number of tile types up to translation if there is an equivalence relation $\sim$ on the tiles $T_{i}$ with a finite number of equivalence classes and $T_{i} \sim T_{j}$ implies that $T_{j}$ is a translate of $T_{i}$. We denote by $\left[T_{i}\right]$ the equivalence class of tile $T_{i}$ and say that $T_{i}$ is a tile of type [ $\left.T_{i}\right]$.

A patch in a tiling is a finite set of its tiles. Two patches are said to be equivalent if one is a translate of the other, that is, there is a single translation which takes every tile in one patch to an equivalent tile in the other patch. The radius of a patch is the radius of the smallest ball containing the patch. 
A tiling is said to have a finite number of local configurations, or FLC for short, if there are a finite number of equivalence classes of patches, up to translation, of any given radius.

An FLC tiling is repetitive if for all $r>0$, there is an $R>0$ such that every patch of radius $r$ can be found, up to translation, in any ball of radius $R$ in the tiling. This is equivalent to minimality of the orbit closure of the tiling, see, e.g., [17], and was called quasiperiodicity in $[9,23]$.

In an SAT, the $\phi$-image of each tile type is a well-defined collection of translates of tile types. If $T_{i}$ is a tile, we can write $\phi T_{i}=\bigcup_{j}\left(T_{i_{j}}+d_{i_{j}}\right)$, which is a finite interiordisjoint union. This subdivision only depends on the type of tile $T_{i}$, in the sense that equivalent tiles have equivalent subdivisions. In particular, we let $m_{i j}$ be the number of tiles of type $j$ in the subdivision of a tile of type $i$. The matrix $\mathbf{m}=\left(m_{i j}\right)$ is the subdivision matrix, it is a nonegative integer matrix which is primitive: some power is strictly positive (by repetitivity of the tiling). The leading eigenvalue of $\mathbf{m}$ is the volume expansion of the SAT, which therefore must be a real Perron number.

Given an SAT, one can select in each of the tile types a point, called a control point, in such a way that the set $\mathcal{C}$ of the control points of tiles in a tiling is forward invariant under $\phi: \phi \mathcal{C} \subset \mathcal{C}$. This can be accomplished as follows [23] (see also [15, Prop. 1.3]): for each tile type $\left[T_{i}\right]$, select one tile in its image under expansion and subdivision. Let the preimage of this tile be $A\left[T_{i}\right] \subset\left[T_{i}\right]$. Then the sequence $\left[T_{i}\right], A\left[T_{i}\right], A\left(A\left[T_{i}\right]\right), \ldots$ nests down to a single point in $\left[T_{i}\right]$, denoted by $c\left(T_{i}\right)$, which we define to be the control point of $T_{i}$. For a tile $T=T_{i}+x$, we let $c(T)=c\left(T_{i}\right)+x$.

\section{Theorem}

The following theorem is stated in [9].

Theorem 3.1 Let $\phi$ be a diagonalizable (over $\mathbb{C}$ ) expanding linear map on $\mathbb{R}^{n}$, and let $\mathcal{T}$ be a self-affine tiling of $\mathbb{R}^{n}$ with expansion $\phi$. Then

(i) every eigenvalue of $\phi$ is an algebraic integer;

(ii) if $\lambda$ is an eigenvalue of $\phi$ of multiplicity $k$ and $\gamma$ is an algebraic conjugate of $\lambda$, then either $|\gamma|<|\lambda|$, or $\gamma$ is also an eigenvalue of $\phi$ of multiplicity greater than or equal to $k$.

The proof is based on the arguments of Thurston [23] and Kenyon [9], but we fill several gaps in those arguments and provide a great deal more detail. In particular, Lemmas 3.7 and 3.8 have no analogs in $[9,23]$. It should be pointed out that the corresponding parts of [23] and [9] have never appeared in refereed publications but have been widely cited and used in the literature on tilings and tiling dynamical systems.

By appropriate choice of a basis, we can assume that the linear map $\phi$ has the real canonical form, see [6, Theorem 6.4.2]. Since $\phi$ is diagonalizable over $\mathbb{C}$, this means that we have a direct sum decomposition

$$
\mathbb{R}^{n}=\bigoplus_{i=1}^{p} E_{i}
$$


into invariant subspaces associated with eigenvalues $\lambda_{i}$ of $\phi$, where we count eigenvalues, having nonnegative imaginary part, with multiplicities. For a real eigenvalue $\lambda_{i}$, the subspace $E_{i}$ is one-dimensional, and $\left.\phi\right|_{E_{i}}$ acts as multiplication by $\lambda_{i}$. For a nonreal eigenvalue $\lambda_{i}$, the subspace $E_{i}$ is two-dimensional. Identifying it with a complex plane, we get that $\left.\phi\right|_{E_{i}}$ acts as multiplication by the complex number $\lambda_{i}$, in other words, as a composition of a dilation and a rotation. We can define a norm on $\|\cdot\|$ on $\mathbb{R}^{n}$ such that

$$
\|x\|=\max _{i}\left\|x_{i}\right\| \quad \text { for } x=\sum_{i=1}^{p} x_{i}, x_{i} \in E_{i},\left\|\phi x_{i}\right\|=\left|\lambda_{i}\right|\left\|x_{i}\right\|
$$

(here $\left\|x_{i}\right\|$ is just the Euclidean norm on $E_{i}$ in our basis).

Beginning of the proof. Let $\mathcal{C}=\mathcal{C}(\mathcal{T})$ be a set of control points of the tiling $\mathcal{T}$. Recall that $\phi(\mathcal{C}) \subset \mathcal{C}$ by construction. Consider $J=\langle\mathcal{C}\rangle$, the free Abelian group generated by $\mathcal{C}$. It is easy to see that $J$ is finitely generated. Indeed, let

$$
\Psi:=\left\{c\left(T^{\prime}\right)-c(T): T, T^{\prime} \in \mathcal{T}, T \neq T^{\prime}, T \cap T^{\prime} \neq \emptyset\right\} .
$$

The set $\Psi$ is finite by FLC, and $J$ is generated by $\Psi$ and an arbitrary control point (we can get from it to any control point by moving "from neighbor to neighbor"). Let us fix free generators $v_{1}, \ldots, v_{N}$ of $J$. These are vectors in $\mathbb{R}^{n}$; of course, they need not be in $\mathcal{C}$. They span $\mathbb{R}^{n}$, since $\mathcal{C}$ is relatively dense. Note that the choice of the generators is nonunique; in fact, we will need to choose them in a specific way at the end of the proof. However, for now any generators will do. Let $V$ be the matrix $V=\left[v_{1} \ldots v_{N}\right]$. This is an $n \times N$ matrix of rank $n$. By the definition of free generators, for every $\xi \in J$, there exists a unique $a(\xi) \in \mathbb{Z}^{N}$ such that

$$
\xi=\operatorname{Va}(\xi) \text {. }
$$

We call $\xi \mapsto a(\xi)$ the "address map". Observe that

$$
\operatorname{Span}_{\mathbb{R}}\{a(\xi): \xi \in \mathcal{C}\}=\mathbb{R}^{N} .
$$

Indeed, $J$ is generated by $\mathcal{C}$, hence every $v_{j}$ is an integral linear combination of control points, and $a\left(v_{j}\right)$ is the $j$ th unit vector in $\mathbb{R}^{N}$.

Lemma 3.2 The address map is uniformly Lipschitz on $\mathcal{C}$ : there exists $L_{1}>0$ such that

$$
\left\|a(\xi)-a\left(\xi^{\prime}\right)\right\| \leq L_{1}\left\|\xi-\xi^{\prime}\right\| \quad \text { for all } \xi, \xi^{\prime} \in \mathcal{C} .
$$

This lemma is a special case of the implication (i) $\Rightarrow(v)$ in [11, Theorem 2.2]. Note that the address map is usually not even continuous on $J$, since $J$ is not discrete in $\mathbb{R}^{n}$ unless we have a "lattice tiling", whereas the range of the address map is a subset of the integer lattice in $\mathbb{R}^{N}$.

Observe that $\phi \mathcal{C} \subset \mathcal{C}$ implies $\phi J \subset J$, hence there exists an integer $N \times N$ matrix $M$ such that

$$
\phi V=V M .
$$


In other words, we have the commutative diagram (where $i$ indicates the natural inclusion)

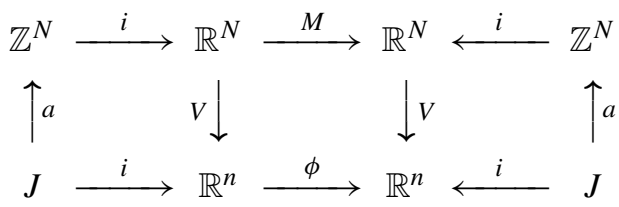

For every (complex) eigenvalue $\lambda$ of $\phi$, we can find a (complex) left eigenvector $e_{\lambda}$ of $\phi$ corresponding to $\lambda$. Then $e_{\lambda} V$ is a left eigenvector for $M$ corresponding to $\lambda$ (note that $e_{\lambda} V \neq 0$ since $V$ has maximal possible rank $n$ ). This proves (i): every eigenvalue of $\phi$ is also an eigenvalue of $M$, hence an algebraic integer. Note also that (7) implies

$$
a(\phi \xi)=M a(\xi), \quad \forall \xi \in J
$$

Lemma 3.3 The matrix $M$ is diagonalizable over $\mathbb{C}$.

Proof Recall that $J$ is a free $\mathbb{Z}$-module, on which $\phi$ acts as an endomorphism, and $M$ is the matrix of this endomorphism in the basis $\mathcal{V}:=\left\{v_{1}, \ldots, v_{N}\right\}$. Note that $\mathbb{Q} \cdot J$ is a vector space over $\mathbb{Q}$, and $\mathcal{V}$ is also a basis of this vector space. Then $\phi$ induces a linear transformation of $\mathbb{Q} \cdot J$, whose matrix in the basis $\mathcal{V}$ is also $M$.

Consider the decomposition (1) of $\mathbb{R}^{n}$ into real eigenspaces $E_{i}$ corresponding to the eigenvalues $\lambda_{i}$ of $\phi$. Decomposing the vectors $v_{j}$ (the generators of $J$ ) in terms of $E_{i}$ yields

$$
J \subset J^{\prime}:=\bigoplus_{i=1}^{p} J_{i} e_{i},
$$

where $e_{i} \in E_{i}$, and $J_{i}$ is a finitely-generated $\mathbb{Z}\left[\lambda_{i}\right]$-module. (Here we identify twodimensional subspaces $E_{i}$ with a complex plane on which $\phi$ acts as multiplication by $\lambda_{i}$.) Then $\mathbb{Q} \cdot J_{i}$ is a vector space over $\mathbb{Q}$ and over $\mathbb{Q}\left(\lambda_{i}\right)$ (a field). Let $\left\{y_{1}^{(i)}, \ldots, y_{r_{i}}^{(i)}\right\}$ be a basis of $\mathbb{Q} \cdot J_{i}$ over $\mathbb{Q}\left(\lambda_{i}\right)$. Let $n_{i}$ be the degree of the algebraic integer $\lambda_{i}$. Then $\left\{\lambda_{i}^{s} y_{k}^{(i)}: 0 \leq s \leq n_{i}-1,1 \leq k \leq r_{i}, i \leq p\right\}$ is a basis for the vector space $\mathbb{Q} \cdot J^{\prime}$ over $\mathbb{Q}$. In this basis, the linear transformation induced by $\phi$ has a block matrix, whose every block is a companion matrix of the minimal polynomial of one of the $\lambda_{i}$ 's. This matrix is diagonalizable over $\mathbb{C}$, since the minimal polynomial has no repeated roots. Finally, we note that the linear transformation induced by $\phi$ on $\mathbb{Q} \cdot J$ is a restriction of the one which is induced on $\mathbb{Q} \cdot J^{\prime}$, hence its matrix, $M$, is diagonalizable as well.

Now suppose that $\gamma$ is a conjugate of $\lambda, \gamma \neq \lambda, \bar{\lambda}$, and $|\gamma|>1$. Then $\gamma$ is an eigenvalue of $M$. Let $U_{\gamma}$ be the (real) eigenspace for $M$ corresponding to $\gamma$. By Lemma 3.3 , there is a projection $\pi_{\gamma}$ from $\mathbb{R}^{N}$ to $U_{\gamma}$ commuting with $M$. By definition, the only eigenvalues of $\left.M\right|_{U_{\gamma}}$ are $\gamma$ and $\bar{\gamma}$ (if $\gamma$ is nonreal). Thus, we can fix a norm on $U_{\gamma}$ satisfying

$$
\|M y\|=|\gamma|\|y\|, \quad y \in U_{\gamma} .
$$


Consider the mapping $f_{\gamma}: \mathcal{C} \rightarrow U_{\gamma}$ given by

$$
f_{\gamma}(\xi)=\pi_{\gamma} a(\xi), \quad \xi \in \mathcal{C} .
$$

We would like to extend $f_{\gamma}$ to the entire space $\mathbb{R}^{n}$. We let

$$
f_{\gamma}\left(\phi^{-k} \xi\right)=M^{-k} f_{\gamma}(\xi), \quad \xi \in \mathcal{C} .
$$

This is well defined, since $M$ is invertible on $U_{\gamma}$, and unambiguous by (8), since $\pi_{\gamma} M=M \pi_{\gamma}$. This way we have $f_{\gamma}$ defined on a dense set

$$
\mathcal{C}_{\infty}:=\bigcup_{k=0}^{\infty} \phi^{-k} \mathcal{C}
$$

Our goal is to show that $f_{\gamma}$ is uniformly continuous on $\mathcal{C}_{\infty}$ and hence can be extended to all of $\mathbb{R}^{n}$. In fact, it is Hölder continuous. Let $\lambda_{\max }$ be the eigenvalue of $\phi$ of maximal modulus. We use the norm (2) on $\mathbb{R}^{n}$. Denote $B_{r}(x)=\left\{y \in \mathbb{R}^{n}\right.$ : $\|y-x\|<r\}$ and let $B_{r}:=B_{r}(0)$.

Lemma 3.4 The map $f_{\gamma}$ is Hölder continuous on $\mathcal{C}_{\infty}$ : there exists $r>0$ and $L_{2}>0$ such that for any $\xi_{1}, \xi_{2} \in \mathcal{C}_{\infty}$ with $\left|\xi_{1}-\xi_{2}\right|<r$, we have

$$
\left\|f_{\gamma}\left(\xi_{1}\right)-f_{\gamma}\left(\xi_{2}\right)\right\| \leq L_{2}\left\|\xi_{1}-\xi_{2}\right\|^{\alpha} \quad \text { for } \alpha=\frac{\log |\gamma|}{\log \left|\lambda_{\max }\right|} .
$$

Proof Let $r>0$ be such that for every $x \in \mathbb{R}^{n}$, the ball $B_{r}(x)$ is covered by a tile containing $x$ and its immediate neighbors; this is possible by FLC. Assume that $\delta=$ $\left\|\xi_{1}-\xi_{2}\right\|<r$ and $\xi_{i}=\phi^{-k} c_{i}$ for some $c_{i} \in \mathcal{C}$ and $k \in \mathbb{N}$. Define $\ell$ to be the smallest positive integer such that

$$
\phi^{k} B_{\delta}\left(\phi^{-k} c_{1}\right) \subset \phi^{\ell} B_{r}\left(\phi^{-\ell} c_{1}\right) .
$$

Since $\ell \leq k$, the last inclusion is equivalent to $\left|\lambda_{\max }\right|^{k-l} \delta \leq r$, so we have

$$
\left|\lambda_{\max }\right|^{-1}(r / \delta) \leq\left|\lambda_{\max }\right|^{k-\ell} \leq r / \delta \text {. }
$$

Observe that

$$
c_{2} \in \phi^{k} \overline{B_{\delta}}\left(\phi^{-k} c_{1}\right) \subset \phi^{\ell} \overline{B_{r}}\left(\phi^{-\ell} c_{1}\right)
$$

so $\phi^{-\ell} c_{1}$ and $\phi^{-\ell} c_{2}$ are in the same or in the neighboring tiles of $\mathcal{T}$ by the choice of $r$. We claim that there exists a finite set $W \subset J$, independent of $c_{1}, c_{2}$, such that

$$
c_{2}-c_{1}=\sum_{i=0}^{\ell} \phi^{i} w_{i}
$$

for some $w_{i} \in W$ (of course, $w_{i}$, as well as $\ell$, depend on $c_{1}, c_{2}$ ). This is standard, but we provide a proof for completeness. 
Let $T_{i} \in \mathcal{T}$ be such that $c_{i}=c\left(T_{i}\right), i=1,2$. By the definition of SAT, there is a (unique) tile $T_{i}^{(1)} \in \mathcal{T}$ such that $\phi T_{i}^{(1)} \supset T_{i}^{(0)}:=T_{i}$. Iterating this, we obtain a sequence of $\mathcal{T}$-tiles $T_{i}^{(j)}$ for $j \geq 0$ such that $\phi T_{i}^{(j)} \supset T_{i}^{(j-1)}$ for $j \geq 1$ and $i=1,2$. Note that $T_{i}^{(\ell)} \supset \phi^{-\ell} T_{i}^{(0)} \ni \phi^{-\ell} c_{i}$, hence $T_{1}^{(\ell)}$ and $T_{2}^{(\ell)}$ either coincide or are adjacent. We have

$$
\begin{aligned}
c_{2}-c_{1}= & \sum_{j=0}^{\ell-1}\left[\left(\phi^{j} c\left(T_{2}^{(j)}\right)-\phi^{j+1} c\left(T_{2}^{(j+1)}\right)\right)-\left(\phi^{j} c\left(T_{1}^{(j)}\right)-\phi^{j+1} c\left(T_{2}^{(j+1)}\right)\right)\right] \\
& +\phi^{\ell} c\left(T_{2}^{(\ell)}\right)-\phi^{\ell} c\left(T_{1}^{(\ell)}\right) .
\end{aligned}
$$

This implies (14), since the set

$$
\left\{c\left(T^{\prime}\right)-\phi c\left(T^{\prime \prime}\right): T^{\prime}, T^{\prime \prime} \in \mathcal{T}, T^{\prime} \subset \phi T^{\prime \prime}\right\}
$$

is finite by FLC, as well as the set $\Psi$ from (3), to which $w_{\ell}$ belongs.

Now we can write, using (3), the additivity of the address map on $J$, and (8),

$$
\begin{aligned}
f_{\gamma}\left(c_{1}\right)-f_{\gamma}\left(c_{2}\right) & =\pi_{\gamma} a\left(c_{2}-c_{1}\right) \\
& =\pi_{\gamma} a\left(\sum_{i=0}^{\ell} \phi^{i} w_{i}\right) \\
& =\sum_{i=0}^{\ell} M^{i} \pi_{\gamma} a\left(w_{i}\right) .
\end{aligned}
$$

Thus, in view of (11) and (9),

$$
\begin{aligned}
\left\|f_{\gamma}\left(\phi^{-k} c_{2}\right)-f_{\gamma}\left(\phi^{-k} c_{1}\right)\right\| & =\left\|M^{-k}\left(f_{\gamma}\left(c_{1}\right)-f_{\gamma}\left(c_{2}\right)\right)\right\| \\
& =|\gamma|^{-k}\left\|f_{\gamma}\left(c_{1}\right)-f_{\gamma}\left(c_{2}\right)\right\| \\
& =|\gamma|^{-k}\left\|\sum_{i=0}^{\ell} M^{i} \pi_{\gamma} a\left(w_{i}\right)\right\| \\
& \leq|\gamma|^{-k} \sum_{i=0}^{\ell}|\gamma|^{i}\left\|\pi_{\gamma} a\left(w_{i}\right)\right\| \leq L^{\prime}|\gamma|^{\ell-k},
\end{aligned}
$$

where $L^{\prime}=\frac{|\gamma|}{|\gamma|-1} \max _{w \in W}\|a(w)\|$. In view of (13),

$$
|\gamma|^{\ell-k}=\left(\left|\lambda_{\max }\right|^{\ell-k}\right)^{\alpha} \leq\left(\left|\lambda_{\max }\right| \delta / r\right)^{\alpha}=\mathrm{const} \cdot\left\|\xi_{1}-\xi_{2}\right\|^{\alpha},
$$

so we obtain the desired inequality. that

Now we extend $f_{\gamma}$ by continuity and obtain a function $f_{\gamma}: \mathbb{R}^{n} \rightarrow U_{\gamma}$. Observe

$$
f_{\gamma} \circ \phi=M \circ f_{\gamma}
$$


since this holds on the dense set $\mathcal{C}_{\infty}$. We also have the following property.

Lemma 3.5 Let $E_{\theta}$ be the real invariant subspace of $\phi$ corresponding to an eigenvalue $\theta$ and suppose that $|\gamma| \geq|\theta|$. Then $\left.f_{\gamma}\right|_{E_{\theta}+x}$ is Lipschitz for any $x \in \mathbb{R}^{n}$, with a uniform constant $2 L_{1}$ (where $L_{1}$ is the constant in Lemma 3.2). If $|\gamma|>|\theta|$, then $\left.f_{\gamma}\right|_{E_{\theta}+x}$ is constant for any $x \in \mathbb{R}^{n}$.

Proof Let $\xi_{1}, \xi_{2} \in \mathbb{R}^{n}$ be such that $\xi_{2}-\xi_{1} \in E_{\theta}$. By (15), we have for $k \in \mathbb{N}$,

$$
\begin{aligned}
\left\|f_{\gamma}\left(\xi_{1}\right)-f_{\gamma}\left(\xi_{2}\right)\right\| & =\left\|M^{-k}\left(f_{\gamma}\left(\phi^{k} \xi_{1}\right)-f_{\gamma}\left(\phi^{k} \xi_{2}\right)\right)\right\| \\
& =|\gamma|^{-k}\left\|f_{\gamma}\left(\phi^{k} \xi_{1}\right)-f_{\gamma}\left(\phi^{k} \xi_{2}\right)\right\| .
\end{aligned}
$$

Let $c_{i}$ be a nearest control point to $\phi^{k} \xi_{i}$; its distance to $\phi^{k} \xi_{i}$ is at most $d_{\max }=$ $\max \{\operatorname{diam}(T): T \in \mathcal{T}\}$. If $k$ is so large that $\left\|\phi^{k} \xi_{1}-\phi^{k} \xi_{2}\right\|>2 d_{\max }$, then $\left\|c_{1}-c_{2}\right\|<$ $2\left\|\phi^{k} \xi_{1}-\phi^{k} \xi_{2}\right\|$, and we have by uniform continuity of $f_{\gamma}$, Lemma 3.2, and (2), with a uniform constant $C_{3}$ :

$$
\begin{aligned}
\left\|f_{\gamma}\left(\phi^{k} \xi_{1}\right)-f_{\gamma}\left(\phi^{k} \xi_{2}\right)\right\| & \leq C_{3}+\left\|f\left(c_{1}\right)-f\left(c_{2}\right)\right\| \\
& \leq C_{3}+L_{1}\left\|c_{1}-c_{2}\right\| \\
& \leq C_{3}+2 L_{1}\left\|\phi^{k} \xi_{1}-\phi^{k} \xi_{2}\right\| \\
& =C_{3}+2 L_{1}|\theta|^{k}\left\|\xi_{1}-\xi_{2}\right\| .
\end{aligned}
$$

Thus,

$$
\left\|f_{\gamma}\left(\xi_{1}\right)-f_{\gamma}\left(\xi_{2}\right)\right\| \leq C_{3}|\gamma|^{-k}+2 L_{1}(|\theta| /|\gamma|)^{k}\left\|\xi_{1}-\xi_{2}\right\| .
$$

The lemma follows by letting $k \rightarrow \infty$. (Recall that $|\gamma| \geq|\theta|>1$.)

Lemma 3.6 The function $f_{\gamma}$ depends only on the tile type in $\mathcal{T}$ up to an additive constant: if $T, T+x \in \mathcal{T}$ and $\xi \in T$, then

$$
f_{\gamma}(\xi+x)=f_{\gamma}(\xi)+\pi_{\gamma} a(x) .
$$

Observe that $x \in \mathcal{C}-\mathcal{C}$, so $a(x)$ is defined, but we cannot write $\pi_{\gamma} a(x)=f_{\gamma}(x)$, since we do not necessarily have $x \in \mathcal{C}$.

Proof It is enough to check (16) on a dense set. Suppose that $\xi=\phi^{-k} c(S) \in T$ for some $S \in \mathcal{T}$. Then $S \subset \phi^{k} T$ and $S+\phi^{k} x \subset \phi^{k}(T+x)$, so $S+\phi^{k} x \in \mathcal{T}$. Thus,

$$
\begin{aligned}
f_{\gamma}(\xi+x) & =f_{\gamma}\left(\phi^{-k} c(S)+x\right) \\
& =f_{\gamma}\left(\phi^{-k} c\left(S+\phi^{k} x\right)\right) \\
& =M^{-k} f_{\gamma}\left(c\left(S+\phi^{k} x\right)\right) \\
& =M^{-k} f_{\gamma}(c(S))+M^{-k} \pi_{\gamma} a\left(\phi^{k} x\right) \\
& =f_{\gamma}(\xi)+\pi_{\gamma} a(x),
\end{aligned}
$$

as desired. Here we used the definition of $f_{\gamma}$ on $\mathcal{C}$ and (8). 
Lemma 3.7 If $|\gamma| \geq|\lambda|$, then $\left.f_{\gamma}\right|_{E_{\lambda}+x}$ is a constant function for any $x \in \mathbb{R}^{n}$.

Proof By Lemma 3.5, this holds if $|\gamma|>|\lambda|$, so it remains to consider the case $|\gamma|=$ $|\lambda|$. We know that for all $x \in \mathbb{R}^{n}$, the restriction $\left.f_{\gamma}\right|_{E_{\lambda}+x}$ is Lipschitz, hence a.e. differentiable by Rademacher's Theorem. It follows that

$$
D(x) u:=\lim _{t \rightarrow 0} \frac{f_{\gamma}(x+t u)-f_{\gamma}(x)}{t}
$$

exists for a.e. $x \in \mathbb{R}^{n}$ for all $u \in E_{\lambda}$ and is a linear transformation in $u$ (from $E_{\lambda}$ to $U_{\gamma}$ ). Moreover, $D(x)$ is measurable in $x$, since it is a limit of continuous functions. Since $D(x)$ is the total derivative, we have

$$
\lim _{k \rightarrow \infty}\left(\sup _{u \in E_{\lambda}, 0<\|u\|<1 / k} \frac{\left\|f_{\gamma}(x+u)-f_{\gamma}(x)-D(x) u\right\|}{\|u\|}\right)=0 \quad \text { for a.e. } x \in \mathbb{R}^{n} .
$$

The functions in parentheses are measurable and converge a.e., hence by Egorov's Theorem they converge uniformly on a set of positive measure. Uniform convergence means that there exists a sequence of positive integers $N_{k} \uparrow \infty$ such that

$$
\begin{gathered}
\Omega:=\left\{\xi \in \mathbb{R}^{n}:\left\|f_{\gamma}(\xi+u)-f_{\gamma}(\xi)-D(\xi) u\right\| \leq\|u\| / k\right. \\
\left.\forall u \in B_{1 / N_{k}} \cap E_{\lambda}, \text { for all } k \text { sufficiently large }\right\}
\end{gathered}
$$

has positive Lebesgue measure. We claim that $\Omega$ has full Lebesgue measure.

Observe that if $T, T+x \in \mathcal{T}$ and $\xi \in T^{\circ}$, then

$$
\xi \in \Omega \Rightarrow \xi+x \in \Omega
$$

by Lemma 3.6. Furthermore, by (15) we have $D(\phi \xi)=M D(\xi) \phi^{-1}$ and, denoting $v=\phi u$, for all $v \in B_{|\lambda| / N_{k}} \cap E_{\lambda}$,

$$
\begin{aligned}
\left\|f_{\gamma}(\phi \xi+v)-f_{\gamma}(\phi \xi)-D(\phi \xi) v\right\| & =\left\|M\left(f_{\gamma}(\xi+u)-f_{\gamma}(\xi)\right)-D(\xi) u\right\| \\
& =|\gamma| \cdot\left\|f_{\gamma}(\xi+u)-f_{\gamma}(\xi)-D(\xi) u\right\| \\
& \leq|\gamma| \cdot\|u\| / k=|\lambda| \cdot\|u\| / k=\|v\| / k,
\end{aligned}
$$

where we used that $\left.\phi\right|_{E_{\lambda}}$ expands the norm by a factor of $|\lambda|$. This shows that $\phi(\Omega) \subset \Omega$.

We will need a version of Lebesgue-Vitali Density Theorem where the differentiation basis is not the set of balls but rather the collection of sets of the form $\phi^{-k} B_{1}$, $k \geq 0$, and their translates. It is a well-known fact in Harmonic Analysis that such sets form a density basis, for any expanding linear map $\phi$ (even nondiagonalizable), see [22, pp. 8-13] or [16, pp. 11-14]. Let $y$ be a density point of $\Omega$, i.e., denoting the Lebesgue measure by $m$,

$$
m\left(\Omega \cap \phi^{-k} B_{1}\left(\phi^{k} y\right)\right) \geq\left(1-\varepsilon_{k}\right) m\left(\phi^{-k} B_{1}\right) \quad \text { for some } \varepsilon_{k} \rightarrow 0 .
$$


Denote by $\left[B_{1}(x)\right]^{\mathcal{T}}$ the patch consisting of those tiles which intersect $B_{1}(x)$. By repetitivity, there exists $R>0$ such that $B_{R}$ contains a translate of $\left[B_{1}(x)\right]^{\mathcal{T}}$ for every $x \in \mathbb{R}^{n}$. Let $y_{k} \in B_{R}$ be such that $\left[B_{1}\left(y_{k}\right)\right]^{\mathcal{T}}$ is a translate of $\left[B_{1}\left(\phi^{k} y\right)\right]^{\mathcal{T}}$. Then

$$
\begin{aligned}
m\left(\Omega \cap B_{1}\left(y_{k}\right)\right) & =m\left(\Omega \cap B_{1}\left(\phi^{k} y\right)\right) \\
& \geq m\left(\phi^{k} \Omega \cap B_{1}\left(\phi^{k} y\right)\right) \\
& =|\operatorname{det} \phi|^{k} m\left(\Omega \cap \phi^{-k} B_{1}\left(\phi^{k} y\right)\right) \\
& \geq|\operatorname{det} \phi|^{k}\left(1-\varepsilon_{k}\right) m\left(\phi^{-k} B_{1}\right)=\left(1-\varepsilon_{k}\right) m\left(B_{1}\right) .
\end{aligned}
$$

We used (18) and $\phi^{k} \Omega \subset \Omega$ in the first two displayed lines above. Let $y^{\prime}$ be a limit point of $y_{k}$. Then we have $m\left(\Omega \cap B_{1}\left(y^{\prime}\right)\right)=m\left(B_{1}\right)$. Thus, $\Omega$ is a set of full measure in $B_{1}\left(y^{\prime}\right)$, and by expansion and translation we conclude that $\Omega$ has full measure in $\mathbb{R}^{n}$, completing the proof of the claim.

Now choose $\ell_{k}$ so that $|\lambda|^{\ell_{k}}>N_{k}$. We have

$$
\begin{aligned}
& \zeta \in \phi^{\ell_{k}} \Omega \Rightarrow\left\|f_{\gamma}(\zeta+v)-f_{\gamma}(\zeta)-D(\zeta) v\right\| \leq\|v\| / k \\
& \quad \text { for all } v \in \phi^{\ell_{k}}\left(B_{1 / N_{k}} \cap E_{\lambda}\right) \supset B_{1} \cap E_{\lambda} .
\end{aligned}
$$

We know that $\Omega^{\prime}=\bigcap_{k>1} \phi^{\ell_{k}} \Omega$ has full measure, hence it is dense. For any $\xi \in \mathbb{R}^{n}$, choose a sequence $\xi_{k} \rightarrow \xi$ such that $D\left(\xi_{k}\right)$ converges (this is possible since $\|D(\xi)\| \leq$ $2 L_{1}$ by Lemma 3.5). Passing to the limit, we obtain that

$$
f_{\gamma}(\xi+v)=f_{\gamma}(\xi)+D(\xi) v \quad \text { for all } v \in B_{1} \cap E_{\lambda} .
$$

This shows that $f$ is affine linear on every $E_{\lambda}$ slice:

$$
f_{\gamma}(\xi+v)=f_{\gamma}(\xi)+D(\xi) v \text { for all } v \in E_{\lambda},
$$

and $D(\xi)=D\left(\xi^{\prime}\right)$ whenever $\xi^{\prime}-\xi \in E_{\lambda}$. Taking $\xi=0$, we see that $\left.f_{\gamma}\right|_{E_{\lambda}}$ is linear. It intertwines $\left.\phi\right|_{E_{\lambda}}$ and $\left.M\right|_{U_{\gamma}}$. But $\{\gamma, \bar{\gamma}\} \cap\{\lambda, \bar{\lambda}\}=\emptyset$, which are the eigenvalues of $\left.\phi\right|_{E_{\lambda}}$ and $\left.M\right|_{U_{\gamma}}$ respectively, hence the only possibility is $\left.f_{\gamma}\right|_{E_{\lambda}} \equiv 0$. Since $f_{\gamma}$ is uniformly continuous on $\mathbb{R}^{n}$ and $\left.f_{\gamma}\right|_{x+E_{\lambda}}$ is affine linear, we obtain that $\left.f_{\gamma}\right|_{x+E_{\lambda}} \equiv$ $\operatorname{const}(x)$.

To motivate the conclusion of the proof, we start with a heuristic discussion. Assume that $|\gamma| \geq|\lambda|$ for the rest of the proof. So far, we have proved that $f_{\gamma}$ is affine linear on the slices $x+E_{\lambda}$. Suppose that we could show that $f_{\gamma}$ is linear on $\mathbb{R}^{n}$. Then we could conclude as follows: $f_{\gamma} \circ \phi=M \circ f_{\gamma}$ and $f_{\gamma}\left(\mathbb{R}^{n}\right)=U_{\gamma}$ (the latter follows from (5) and the definition of $f_{\gamma}$ ) would imply that $\phi$ restricted to a linear subspace and $\left.M\right|_{U_{\gamma}}$ are linearly conjugate:

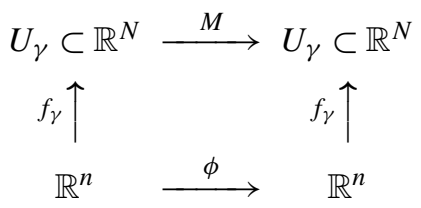


and hence $\gamma$ is an eigenvalue of $\phi$ of multiplicity at least $\operatorname{dim} U_{\gamma} \geq \operatorname{dim} E_{\lambda}$, as desired.

This scheme does work, but with some modifications. We are able to show that $f_{\gamma}$ is affine linear in some, but possibly not all, directions complementary to $E_{\lambda}$. It is linear in directions for which the differences between control points for tiles of the same type project densely.

Let $\Xi=\Xi(\mathcal{T})$ denote the set of translation vectors between tiles of the same type, and let $P_{\lambda}$ be the projection from $\mathbb{R}^{n}$ to $E_{\lambda}$ commuting with $\phi$ (note that the projection $\pi_{\gamma}$ acts in another space, $\mathbb{R}^{N}$ ).

Consider the set $\left(I-P_{\lambda}\right) \Xi$, that is, the projection of $\Xi$ onto the other eigenspaces of $\phi$. This projection may look like a lattice in some directions and fail to be discrete in other directions. We consider the directions in which this set is not discrete, more precisely, those directions in which there are arbitrarily small nonzero vectors in $(I-$ $\left.P_{\lambda}\right) \Xi$, and denote the span of these directions $E^{\prime}$. What we will prove is that $f_{\gamma}$ is affine linear on all $E^{\prime}$ slices and hence on all $E^{\prime} \oplus E_{\lambda}$ slices. We will then show that the subspace $E:=E^{\prime} \oplus E_{\lambda}$ is $\phi$-invariant and is spanned by the vectors of $\Xi$ contained in it. This will allow us to essentially restrict the entire construction to $\left.\phi\right|_{E}$ and conclude as indicated above, using that $\left.f_{\gamma}\right|_{E}$ is linear.

Now let us be more formal and for each $\varepsilon>0$, define $E_{\varepsilon} \subset \mathbb{R}^{n}$ to be the subspace

$$
E_{\varepsilon}=\operatorname{Span}_{\mathbb{R}}\left(B_{\varepsilon} \cap\left(I-P_{\lambda}\right) \Xi\right) \subset E_{\lambda}^{\perp} \subset \mathbb{R}^{n},
$$

where $E_{\lambda}^{\perp}$ is the $\phi$-invariant subspace complementary to $E_{\lambda}$. Further, consider

$$
E^{\prime}:=\bigcap_{\varepsilon>0} E_{\varepsilon}
$$

We have $\phi \Xi \subset \Xi$ and $P_{\lambda} \phi=\phi P_{\lambda}$, and hence

$$
\phi\left(\left(I-P_{\lambda}\right) \Xi\right) \subset\left(I-P_{\lambda}\right) \Xi .
$$

Note that $E_{\varepsilon}$ are decreasing linear subspaces of $E_{\lambda}^{\perp} \subset \mathbb{R}^{n}$, hence $E^{\prime}=E_{\varepsilon}$ for some $\varepsilon>0$, and so $E^{\prime}=E_{\varepsilon^{\prime}}$ for all $0<\varepsilon^{\prime} \leq \varepsilon$. Since $\phi E_{\varepsilon^{\prime}} \subset E_{c \varepsilon^{\prime}}$ for $c=\|\phi\|$, we see that $E^{\prime}$ is $\phi$-invariant. We then define

$$
E:=E^{\prime}+E_{\lambda}
$$

Lemma $\left.3.8 f_{\gamma}\right|_{E+x}$ is affine linear for every $x \in \mathbb{R}^{n}$.

Proof Choose $\varepsilon$ so that $E^{\prime}=E_{\varepsilon}$. Let $\varepsilon^{\prime}<\varepsilon$ and define

$$
E^{\prime \prime}:=\operatorname{Span}\left(B_{\varepsilon^{\prime}} \cap\left(I-P_{\lambda}\right)\left(\mathcal{C}_{1}-\mathcal{C}_{1}\right)\right)
$$

where $\mathcal{C}_{1}$ is the set of control points of tiles of type 1 (of course, we could equally well choose another tile type). First, we claim that

$$
E^{\prime}=E^{\prime \prime} .
$$


Indeed, $\mathcal{C}_{1}-\mathcal{C}_{1} \subset \Xi$, hence $E^{\prime \prime} \subset E^{\prime}$. Choose $\ell$ so large that $\phi^{\ell} \Xi \subset \mathcal{C}_{1}-\mathcal{C}_{1}$; such an $\ell$ exists by primitivity of the tile substitution (the $\ell$ th power of the substitution of any tile contains tiles of all types). We then have

$$
E^{\prime}=\phi^{\ell} E^{\prime}=\phi^{\ell} E_{\varepsilon^{\prime} /\|\phi\|^{\ell}} \subset \operatorname{Span}\left(B_{\varepsilon^{\prime}} \cap\left(I-P_{\lambda}\right) \phi^{\ell} \Xi\right) \subset E^{\prime \prime} .
$$

The claim is proved.

Now suppose $x \in \mathcal{C}_{1}-\mathcal{C}_{1}$, so there exists $T \in \mathcal{T}$ of type 1 such that $T+x \in \mathcal{T}$. By Lemma 3.6,

$$
\xi \in T \Rightarrow f_{\gamma}(\xi+x)=f_{\gamma}(\xi)+\pi_{\gamma} a(x) .
$$

But Lemma 3.7 implies that $f_{\gamma}(\xi+x)=f_{\gamma}\left(\xi+x-P_{\lambda} x\right)$, so

$$
f_{\gamma}\left(\xi+\left(I-P_{\lambda}\right) x\right)=f_{\gamma}(\xi)+\pi_{\gamma} a(x) \text { for } \xi \in T .
$$

We want to show that $f_{\gamma}$ is affine linear on all $E$-slices. Since $f_{\gamma}$ is constant on all $E_{\lambda}$-slices by Lemma 3.7, it is enough to verify that $f_{\gamma}$ is affine linear on all $E^{\prime}$-slices (recall that $E=E^{\prime}+E_{\lambda}$ ). Fix a small $\varepsilon^{\prime}$ as in (19) and select a basis of $E^{\prime}$ of the form $y_{i}=\left(I-P_{\lambda}\right) x_{i} \in B_{\varepsilon^{\prime}}$, with $x_{i} \in \mathcal{C}_{1}-\mathcal{C}_{1}$, for $i=1, \ldots, \operatorname{dim} E^{\prime}$. Now for any $\xi$ in the interior of $T$ such that $B_{r}(\xi) \subset T$, we obtain from (20):

$$
f_{\gamma}\left(\xi+\sum_{i} b_{i} y_{i}\right)=f_{\gamma}(\xi)+\sum_{i} b_{i} \pi_{\gamma} a\left(x_{i}\right)
$$

for all $b_{i} \in \mathbb{Z}$ such that $\sum_{i} b_{i} y_{i} \in B_{r}$. (Here we should note that, in view of Lemma 3.6, (20) transfers to all tiles equivalent to $T$. Since all the $x_{i}$ are translates between two copies of $T$, we can apply the equality for any $x_{i}$ in any of the translates.) This shows that $f_{\gamma}$ is affine linear on a large chunk of the lattice in $E^{\prime}$ generated by small vectors $y_{i}$, translated in such a way that $\xi$ becomes the origin. It is an easy exercise to pass to the limit as $\varepsilon^{\prime} \rightarrow 0$ and conclude that $f_{\gamma}$ is affine linear in the $E^{\prime}$-direction on $B_{r}(\xi) \cap\left(E^{\prime}+\xi\right)$. To be a bit more precise, we can verify that

$$
f_{\gamma}\left(\frac{\zeta_{1}+\zeta_{2}}{2}\right)=\frac{f_{\gamma}\left(\zeta_{1}\right)+f_{\gamma}\left(\zeta_{2}\right)}{2} \quad \text { for all } \zeta_{1}, \zeta_{2} \in B_{r}(\xi) \cap\left(E^{\prime}+\xi\right)
$$

Since $f_{\gamma}$ is continuous, this implies that

$$
f_{\gamma}(\zeta)=A_{\xi} \zeta+b_{\xi} \quad \text { for all } \zeta \in B_{r}(\xi) \cap\left(E^{\prime}+\xi\right),
$$

see, e.g., [1, 2.1.4], where it is called the "Jensen functional equation". The details are straightforward.

Since (22) holds on all slices of $T$, by "expanding and translating" with the help of (15) and Lemma 3.6, we obtain the claim of the lemma.

\section{Lemma 3.9}

$$
E=\operatorname{Span}_{\mathbb{R}}((\mathcal{C}-\mathcal{C}) \cap E) .
$$


Proof Denote $W:=\operatorname{Span}_{\mathbb{R}}((\mathcal{C}-\mathcal{C}) \cap E)$. First, we show that $E_{\lambda} \subset W$. Let $w \in E_{\lambda}$. The set $\mathcal{C}_{1}$ (control points of type-1 tiles) is relatively dense in $\mathbb{R}^{n}$; let $R>0$ be such that every open ball of radius $R$ hits $\mathcal{C}_{1}$. Let $\xi_{j} \in \mathcal{C}_{1}$ be such that $\left\|\xi_{j}-j w\right\|<R$ for all $j \geq 0$. Then

$$
\left\|\left(I-P_{\lambda}\right) \xi_{j}\right\|=\left\|\left(I-P_{\lambda}\right)\left(\xi_{j}-j w\right)\right\| \leq\left(1+\left\|P_{\lambda}\right\|\right) R, \quad j \geq 0 .
$$

It follows that there exists a sequence of pairs $\left(i_{k}, j_{k}\right)$, with $i_{k}-j_{k} \rightarrow+\infty$, such that

$$
\left\|\left(I-P_{\lambda}\right)\left(\xi_{i_{k}}-\xi_{j_{k}}\right)\right\| \rightarrow 0 \quad \text { as } k \rightarrow \infty .
$$

Therefore, $\left(I-P_{\lambda}\right)\left(\xi_{i_{k}}-\xi_{j_{k}}\right) \in E^{\prime}$ for $k$ sufficiently large, and hence $\xi_{i_{k}}-\xi_{j_{k}} \in E$ for $k \geq k_{0}$. Now,

$$
\left\|\left(\xi_{i_{k}}-\xi_{j_{k}}\right)-w\left(i_{k}-j_{k}\right)\right\| \leq 2 R,
$$

hence $\zeta_{k}:=\left(\xi_{i_{k}}-\xi_{j_{k}}\right) /\left(i_{k}-j_{k}\right) \rightarrow w$. But $\zeta_{k} \in W$ for $k \geq k_{0}$, hence $w \in W$, since $W$ is closed, being a linear subspace of $\mathbb{R}^{n}$.

Now recall that $E^{\prime}$ is spanned by certain vectors of the form $\xi-P_{\lambda} \xi$, with $\xi \in$ $\Xi \subset \mathcal{C}-\mathcal{C}$. Since $P_{\lambda} \xi \in E_{\lambda} \subset E$, we have that these vectors $\xi$ are in $E$, and hence $E^{\prime} \subset W$. This proves that $E=E^{\prime}+E_{\lambda} \subset W$, as desired.

Conclusion of the proof of Theorem 3.1 As mentioned earlier, we would like to run the entire construction essentially restricting ourselves to the subspace $E$, which is $\phi$ invariant, contains $E_{\lambda}$ and is spanned by the vectors of $\mathcal{C}-\mathcal{C}$ in it. We do not literally do this, because it is not clear what the intersection of the tiling with $E$ looks like; rather, we make sure that the construction on $\mathbb{R}^{n}$ is compatible with this subspace structure. Recall that at the beginning of the proof we considered the free Abelian group $J=\langle\mathcal{C}\rangle$ and its free generators $v_{1}, \ldots, v_{N}$. We will now use a more specific choice of the generators. Namely, let

$$
\widetilde{J}:=\langle(\mathcal{C}-\mathcal{C}) \cap E\rangle=\operatorname{Span}_{\mathbb{Z}}((\mathcal{C}-\mathcal{C}) \cap E)
$$

Clearly, $\widetilde{J}$ is an Abelian subgroup of $J$, and $\operatorname{Span}_{\mathbb{R}} \widetilde{J}=E$ by Lemma 3.9. Is it possible to choose the free generators for $J$ as an extension of a set of free generators for $\widetilde{J}$ ? Maybe not, but we can choose $v_{1}, \ldots, v_{N}$, the free generators of $J$, so that $d_{1} v_{1}, \ldots, d_{s} v_{s}$ are free generators of $\widetilde{J}$ for some positive integers $d_{j}$ and $s \leq N$ (see, e.g., [7, Theorem II.1.6]).

Recall that $\phi$ acts on $J$, and on the generators $v_{j}$ this action is given by an integer matrix $M$. Since $\phi$ also acts on $\widetilde{J}$, we claim that

$$
M=\left(\begin{array}{c|c}
\tilde{M} & * \\
\hline 0 & *
\end{array}\right),
$$

where $\tilde{M}$ is an $s \times s$ matrix. Indeed, $\phi\left(v_{i}\right), i \leq N$, is a unique integral linear combination of $\left\{v_{j}\right\}_{j \leq N}$, with the coefficients coming from the $i$ th column of $M$. On the other hand, $\phi\left(d_{i} v_{i}\right), i \leq s$, is an integral linear combination of $\left\{d_{j} v_{j}\right\}_{j \leq s}$, since the 
latter are free generators of $\widetilde{J}$. This implies that $\phi\left(v_{i}\right), i \leq s$, is an integral linear combination of $\left\{d_{j} v_{j}\right\}_{j \leq s}$, that is,

$$
\phi\left[v_{1} \ldots v_{s}\right]=\left[v_{1} \ldots v_{s}\right] \tilde{M}
$$

where $\tilde{M}$ is an integral $s \times s$ matrix. Thus, the matrix $M$ is block upper-triangular, with the upper left corner $\tilde{M}$, as claimed above.

Note that

$$
\operatorname{Span}_{\mathbb{R}}\left(\left\{v_{j}\right\}_{j \leq s}\right)=\operatorname{Span}_{\mathbb{R}}\left(\left\{d_{j} v_{j}\right\}_{j \leq s}\right)=\operatorname{Span}_{\mathbb{R}}((\mathcal{C}-\mathcal{C}) \cap E)=E
$$

by construction. By (23) and (24), there is an $\widetilde{M}$-invariant subspace of $\mathbb{R}^{s}$ on which $\widetilde{M}$ acts isomorphically (linearly conjugate) to $\left.\phi\right|_{E}$. Since $E \supset E_{\lambda}$, we obtain that $\lambda$ is an eigenvalue of $\widetilde{M}$, with the multiplicity greater or equal to $\operatorname{dim} E_{\lambda}$. Because $\gamma$ is an algebraic conjugate of $\lambda$ and $\widetilde{M}$ is an integer matrix, we have that $\gamma$ is also an eigenvalue of $\tilde{M}$, with the multiplicity $\geq \operatorname{dim} E_{\lambda}$. Let $\widetilde{U}_{\gamma}$ be the real invariant subspace of $\widetilde{M}$ corresponding to $\gamma$.

Abusing notation a bit, we will identify $\mathbb{R}^{s}$ with the subspace of $\mathbb{R}^{N}$ generated by the first $s$ coordinates. Then $\widetilde{U}_{\gamma} \subset U_{\gamma}$.

Let $a: J \rightarrow \mathbb{Z}^{N}$ be the address map, as in (4). Then $a(\widetilde{J}) \subset \mathbb{Z}^{s}$ (using a similar abuse of notation, so that $\mathbb{Z}^{s} \subset \mathbb{Z}^{N}$ ). By construction,

$$
\operatorname{Span}_{\mathbb{Z}}\left\{a\left(\xi-\xi^{\prime}\right): \xi, \xi^{\prime} \in \mathcal{C}, \xi-\xi^{\prime} \in E\right\}=\bigoplus_{j=1}^{s} d_{j} \mathbb{Z} \subset \mathbb{Z}^{s}
$$

hence

$$
\operatorname{Span}_{\mathbb{R}}\left\{a\left(\xi-\xi^{\prime}\right): \xi, \xi^{\prime} \in \mathcal{C}, \xi-\xi^{\prime} \in E\right\}=\mathbb{R}^{s}
$$

It follows that

$$
\operatorname{Span}_{\mathbb{R}}\left\{\pi_{\gamma}\left(a(\xi)-a\left(\xi^{\prime}\right)\right): \xi, \xi^{\prime} \in \mathcal{C}, \xi-\xi^{\prime} \in E\right\}=\pi_{\gamma}\left(\mathbb{R}^{s}\right)=\widetilde{U}_{\gamma}
$$

Recall that $f_{\gamma}: \mathbb{R}^{n} \rightarrow \mathbb{R}^{N}$, defined originally by $f_{\gamma}(\xi)=\pi_{\gamma}(a(\xi))$ on control points, is uniformly continuous, $f_{\gamma} \circ \phi=M \circ f_{\gamma}$, and $\left.f_{\gamma}\right|_{E+x}$ is affine linear for all $x$ by Lemma 3.8. Note that $\left.f_{\gamma}\right|_{E}$ is linear, since $f_{\gamma}(0)=0$.

We claim that $f_{\gamma}(E) \supset \widetilde{U}_{\gamma}$. Indeed, every $f_{\gamma}(E+x)$ is a translate of a linear subspace, which must be a translate of $f_{\gamma}(E)$ by the uniform continuity of $f_{\gamma}$. It follows that for $\xi, \xi^{\prime} \in \mathcal{C}, \xi-\xi^{\prime} \in E$,

$$
\pi_{\gamma}\left(a(\xi)-a\left(\xi^{\prime}\right)\right)=f_{\gamma}(\xi)-f_{\gamma}\left(\xi^{\prime}\right) \in f_{\gamma}(E),
$$

whence $\widetilde{U}_{\gamma} \subset f_{\gamma}(E)$ by (25). The claim is verified. 
Since $f_{\gamma}(E)$ contains $\widetilde{U}_{\gamma}$, there exists a $\phi$-invariant subspace $\widetilde{E} \subset E \subset \mathbb{R}^{n}$ such that $f_{\gamma}$ maps $\widetilde{E}$ isomorphically onto $\widetilde{U}_{\gamma}$ :

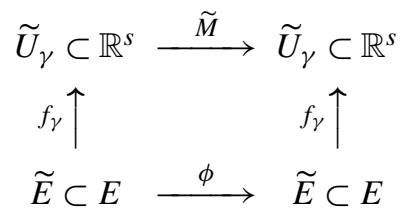

Thus, the linear map $\left.f_{\gamma}\right|_{\widetilde{E}}$ conjugates $\left.\phi\right|_{\widetilde{E}}$ to $\left.\widetilde{M}\right|_{\widetilde{U}_{\gamma}}=\left.M\right|_{\widetilde{U}_{\gamma}}$, hence $\gamma$ is an eigenvalue of $\phi$ of multiplicity $\geq \operatorname{dim} E_{\lambda}$, as desired.

Acknowledgement We are grateful to Misha Lyubich for a suggestion which helped to prove Lemma 3.7.

\section{References}

1. Aczél, J.: Lectures on Functional Equations and Their Applications. Mathematics in Science and Engineering, vol. 19. Academic Press, New York (1966)

2. Barat, G., Berthé, V., Liardet, P., Thuswaldner, J.: Dynamical directions in numeration. Numération, pavages, substitutions. Ann. Inst. Fourier (Grenoble) 56(7), 1987-2092 (2006)

3. Bombieri, E., Taylor, J.E.: Quasicrystals, tilings and algebraic number theory: some preliminary connections. Contemp. Math. 64, 241-264 (1987)

4. Epstein, D., Cannon, J., Holt, D., Levy, S., Paterson, M., Thurston, W.: Word Processing in Groups. Jones and Bartlett, Boston (1992)

5. Furukado, M., Ito, S., Robinson, E.A. Jr.: Tilings associated with non-Pisot matrices. Ann. Inst. Fourier (Grenoble) 56(7), 2391-2435 (2006)

6. Hirsch, M., Smale, S.: Differential Equations, Dynamical Systems, and Linear Algebra. Academic Press, New York (1974)

7. Hungerford, T.W.: Algebra. Graduate Texts in Mathematics, vol. 73. Springer, New York (1980)

8. Kenyon, R.: The construction of self-similar tilings. Geom. Funct. Anal. 6(3), 471-488 (1996)

9. Kenyon, R.: PhD thesis, Princeton University (1990)

10. Kenyon, R., Vershik, A.: Arithmetic construction of sofic partitions of hyperbolic toral automorphisms. Ergod. Theory Dyn. Syst. 18(2), 357-372 (1998)

11. Lagarias, J.: Geometric models for quasicrystals, I: Delone sets of finite type. Discrete Comput. Geom. 21, 161-191 (1999)

12. Lagarias, J.C., Wang, Y.: Self-affine tiles in $R^{n}$. Adv. Math. 121(1), 21-49 (1996)

13. Lind, D.: The entropies of topological Markov shifts and a related class of algebraic integers. Ergod. Theory Dyn. Syst. 4(2), 283-300 (1984)

14. Penrose, R.: Pentaplexity: a class of nonperiodic tilings of the plane. Math. Intel. 2(1), 32-37 (1979)

15. Praggastis, B.: Numeration systems and Markov partitions from self similar tilings. Trans. Am. Math. Soc. 351(8), 3315-3349 (1999)

16. Pugh, C., Shub, M.: Stable ergodicity and julienne quasi-conformality. J. Eur. Math. Soc. 2(1), 1-52 (2000)

17. Radin, C., Wolff, M.: Space tilings and local isomorphism. Geom. Dedic. 42(3), 355-360 (1992)

18. Rauzy, G.: Nombres algébriques et substitutions. Bull. Soc. Math. Fr. 110, 147-178 (1982)

19. Robinson, E.A. Jr.: Symbolic dynamics and tilings of $\mathbb{R}^{d}$. In: Symbolic Dynamics and its Applications. Proc. Sympos. Appl. Math., vol. 60, pp. 81-119. Amer. Math. Soc., Providence (2004)

20. Sinai, Y.: Markov partitions and U-diffeomorphisms. Funktsional. Anal. Prilozhen. 2(1), 64-89 (1968)

21. Solomyak, B.: Dynamics of self-similar tilings. Ergod. Theory Dyn. Syst. 17(3), 695-738 (1997)

22. Stein, E.M.: Harmonic Analysis. Princeton University Press, Princeton (1993)

23. Thurston, W.: AMS Lecture Notes (1989) 